\title{
Rendimento e composição do óleo essencial de patchouli (Pogostemon cablin) conforme o tempo de extração
}

COSTA, G.A. ${ }^{1}$, CARVALHO FILHO, J.L.S. ${ }^{2}$; DESCHAMPS, C. ${ }^{3}$

1 Universidade Federal de Goiás/UFG, Departamento de Agronomia, Campus Jataí/Jatobá, Rodovia BR 364, Km 192, no 3.800 - CEP:75.801-615 - Jataí / GO. Email:andressagiovannini@yahoo.com.br ²Universidade Federal Rural de Pernambuco, Departamento de Agronomia, Rua Dom Manoel de Medeiros, S/N, Dois IrmãosCEP: 52171-900-Recife/PE. Email: joseluiz.ufrpe@yahoo.com.br 3Universidade Federal do Paraná/UFPR, Departamento de Fitotecnia e Fitossanitarismo, Rua dos Funcionários, 1540 , Juvevê - CEP: 80035-050 Curitiba/PR. Email: cicero@ufpr.br

\begin{abstract}
RESUMO: O patchouli possui óleo essencial nas folhas com utilização principalmente na indústria de perfumaria. O objetivo foi avaliar o melhor tempo de extração de óleo essencial de folhas secas de patchouli. Os tratamentos foram 1, 2, 3, 4, 5, 6, 7 e 8 horas de extração, através do método de hidrodestilação, com aparelho graduado do tipo Clevenger e balões com capacidade de $2 \mathrm{~L}$.O delineamento foi inteiramente casualizado com três repetições. O material destilado foi seco à sombra até atingir aproximadamente $20 \%$ de umidade. Para cada tratamento, foram utilizadas amostras de $50 \mathrm{~g}$ de massa seca foliar. Foram avaliados o rendimento e a composição do óleo essencial. Não houve diferença entre os diferentes tempos de extração no rendimento de óleo essencial, podendo a extração do óleo essencial de patchouli ser realizada com uma hora de extração. O tempo de extração aumenta as porcentagens relativas do betaguaieno $(0,81 \%)$, beta-patchouleno $(1,26 \%)$, alfa-selineno $(1,37 \%)$, cariofileno $(2,44 \%)$, alfapatchouleno $(3,08 \%)$ e gama-patchouleno $(4,82 \%)$. O teor de pogostol $(5,11 \%)$ reduz com o aumento do tempo de extração. O patchoulol, alfa-guaieno, alfa-bulneseno e seicheleno não sofrem influencia do tempo de extração.
\end{abstract}

Palavras-chave: Pogostemon cablin, destilação, patchoulol

ABSTRACT: Yield and composition of the patchouli (Pogostemon cablin) essential oil according to the extraction time. Patchouli accumulatea an essential oil on its leaves, and it is mainly used by the perfume industry. The purpose of this work was to determine the distillation time for the extraction of essential oils from the leaves of patchouli. The treatments included 1 , $2,3,4,5,6,7$ and 8 hours of extraction through the hydrodistillation method, using a Clevenger apparatus. The experimental design was completely randomized with three replications. The leaves were dried at room temperature until they reached $20 \%$ of humidity. The essential oil yield was measured from samples containing $50 \mathrm{~g}$ of leaf dry mass. The essential oil production and composition were evaluated. No differences among the treatments were found on the essential oil yield, suggesting that the essential oil extraction can be carried out for 1 hour according to the experimental conditions. However, extending the extraction time, an increase in the relative percentage of beta-patchoulene (1.26\%), cariofilene $(2.44 \%)$, gamma-patchoulene $(4.82 \%)$, alpha-patchoulene $(3.08 \%)$, beta-guaiene $(0.81 \%)$ and alpha-selinene $(1.37 \%)$ was observed. The pogostol $(5.11 \%)$ content was reduced with the increase of the extraction time. Patchoulol, alpha-guaieno, alpha-bulneseno and seicheleno contents were not affected by the evaluated extraction times.

Keywords: Pogostemon cablin, hydrodestillation, patchoulol

\section{INTRODUÇÃO}

O patchouli, Pogostemon cablin Benth. (Lamiaceae), é conhecido pelo óleo essencial extraído de suas folhas, amplamente empregado em perfumaria e cosmética, como base e característica duradoura às fragrâncias, e como matéria-prima muito importante na fabricação de sabonetes,

Recebido para publicação em 24/10/2011

Aceito para publicação em 13/09/2012

Rev. Bras. PI. Med., Campinas, v.15, n.3, p.319-324, 2013. 
cosméticos, incensos, produtos de higiene oral e pós-barba (Milchard et al., 2004; Singh et al., 2002). São também conhecidas atividades antibacteriana (Khare, 2007), antioxidante (Wei \& Shibamoto, 2007), inseticida (Pavela, 2005), e repelente contra insetos (Salerno et al., 2004). Atualmente esta entre os 18 óleos essenciais com maior importância comercial no mundo, e no Brasil a indústria Raros produz, beneficia e exporta $12 \mathrm{t}$ ano $^{-1}$ deste óleo essencial (Bizzo et al., 2009)

O principal responsável por estas características e pela nota típica de seu óleo essencial é o patchoulol ou álcool patchouli, um sesquiterpeno oxigenado. O óleo ainda contém um grande número de outros hidrocarbonetos sesquiterpenos, em torno de 27 compostos, tais como, $\alpha, \sigma, \beta$-patchoulenos, $\alpha$-bulneseno, $\alpha$-guaieno e seicheleno, com estruturas claramente relacionadas ao patchoulol e outros sesquiterpenos (Wei \& Shibamoto, 2007; Deguerry et al., 2006; Hu et al., 2006; Buré \& Sellier, 2004; Singh et al., 2002).

Alguns aspectos influenciam a produção de óleos essenciais, como os fatores genéticos e ambientais, e outros influenciam no rendimento, como o método e o tempo de extração. Para a extração de óleo essencial podem ser aplicados diversos métodos, como a hidrodestilação, maceração, extração por solvente, enfloração, gases supercríticos e micro-ondas. Dentre esses, o de maior aplicação é o de hidrodestilação (Craveiro et al., 1981). A extração do óleo essencial de patchouli, de acordo com Joy et al. (2001) deve ser pelo método de destilação por arraste a vapor, relatando o fato de haver maior rendimento neste processo. Ainda segundo estes autores o tempo de destilação recomendado para o patchouli é de seis a oito horas.

O tempo de extração varia de acordo com a espécie, local e estrutura especializada onde se encontra o óleo essencial. Fatores de pós-colheita, como secagem e processamento do material, também influenciam no rendimento e qualidade do óleo essencial. Ehlert et al. (2006), avaliaram o tempo de hidrodestilação na extração do óleo essencial de sete espécies medicinais, verificando que cada uma das espécies apresentou rendimento diferente em relação ao tempo de hidrodestilação, havendo uma variação entre 130 a 260 min Para a espécie Mentha $x$ Piperita o tempo de extração do óleo essencial apresentou grande variação (60 a $150 \mathrm{~min}$ ) (Souza et al., 2006; Valmorbida et al., 2006), sugerindo a importância de uma avaliação prévia do tempo de extração do óleo essencial para cada espécie, levando em consideração as particularidades de método e aparelho de extração utilizados, visando-se obter a otimização do processo.
Com base nas informações, o objetivo deste trabalhor foi avaliar o efeito de diferentes tempos de extração no rendimento e composição do óleo essencial de patchouli em hidrodestilação.

\section{MATERIAIS E MÉTODOS}

O trabalho foi desenvolvido no Laboratório de Ecofisiologia do Departamento de Fitotecnia e Fitossanitarismo - UFPR. O material vegetal utilizado constituiu-se de folhas de $P$. cablin Benth, fornecidas pela empresa Hérbia Beneficiamento de produtos LTDA, situada no município de JoinvilleSC, coletadas no mês de abril de 2006.

O delineamento experimental foi inteiramente casualizado, sendo comparados os tempos de extração de 1, 2, 3, 4, 5, 6, 7 e 8 horas, após iniciar a fervura, com três repetições, sendo cada repetição constituída de uma planta. $O$ aparelho de extração utilizado foi do tipo Clevenger com dedo frio, para óleos mais densos que a água, com graduação para $10 \mathrm{~mL}$ e manta aquecedora para balão de vidro com capacidade de $2 \mathrm{~L}$. Ao término de cada período de extração, foram avaliados o rendimento e a composição dos óleo essencial coletados.

O material utilizado para extração foi desidratado à sombra até atingir, aproximadamente, $20 \%$ de umidade. O controle da secagem foi determinado pelo peso túrgido de folhas amostradas do material vegetal.

Para a destilação foram utilizadas amostras de $50 \mathrm{~g}$ de massa seca foliar em $1 \mathrm{~L}$ de água destilada. Após a extração, as amostras foram armazenadas em congelador, onde permaneceram até o momento da análise. Foram separados $20 \mathrm{~g}$ do material para determinação do teor de umidade no momento da extração, pela secagem em estufa com circulação de ar forçada a $65^{\circ} \mathrm{C}$ até obter massa constante.

As análises dos constituintes químicos do óleo essencial foram realizadas por meio de cromatografia a gás acoplada à espectrometria de massas no Departamento de Engenharia Química da UFPR. O cromatógrafo utilizado foi da marca Varian Inc. (modelo CP-3800), com detector Saturn $2000 \mathrm{MS} / \mathrm{MS}$ e coluna sílica fundida com $100 \mathrm{~m}$ de comprimento (fase estacionária PONA). Foi utilizado hélio como gás de arraste sob pressão da coluna de 49,5 psi. A condição inicial de temperatura foi de $120^{\circ} \mathrm{C}$ durante 22 minutos, com posterior elevação para $230^{\circ} \mathrm{C}$ durante 20 minutos com razão de aquecimento de $10^{\circ} \mathrm{C}$ por minuto. $\mathrm{O}$ volume de $0,2 \mu \mathrm{L}$ de óleo essencial injetado com razão de split 200 e temperatura de injeção de $200{ }^{\circ} \mathrm{C}$. A identificação dos constituintes químicos foi realizada comparando-se à biblioteca da Nist 98 (Varian Inc.).

Rev. Bras. PI. Med., Campinas, v.15, n.3, p.319-324, 2013. 
As médias foram testadas quanto à sua homogeneidade pelo teste de Bartlett. Os dados foram submetidos à análise de variância e em caso de haver diferença significativa entre os tratamentos pelo teste de $F(P \leq 0,05)$, as médias dos tratamentos foram comparadas por regressão polinomial e pelo teste de Tukey a $5 \%$ de probabilidade.

\section{RESULTADOS E DISCUSSÃO}

Não houve diferença entre os diferentes tempos de extração no rendimento do óleo essencial de patchouli pelo teste de $\mathrm{F}(\mathrm{P}<0,05 ; \mathrm{CV}=24,99 \%)$, sendo o maior valor obtido com sete horas de extração $\left(56,3 \mu \mathrm{L} \mathrm{g}^{-1}\right)$ (Figura 1).

Observou-se que apesar não ter apresentado diferença significativa na análise de regressão, há uma tendência do aumento no rendimento de óleo essencial com o aumento no tempo de extração (Figura 1).

Prins et al. (2006) também não observaram o efeito significativo do tempo de hidrodestilação (30, 60, 90 e 120 minutos) no rendimento de óleo essencial de alecrim (Rosmarinus officinalis). Porém, em pimenta rosa (Schinus molle) o rendimento de óleo essencial foi diretamente proporcional ao tempo de extração (1, 2, 4 e 6 horas), sendo a maior média $(4,61 \%)$ obtida com quatro horas (Silva et al., 2005). Em um ensaio para determinação do tempo (30, 60 e 90 minutos) de máxima extração de óleo essencial de menta (Mentha piperita) demonstrou que após 60 minutos de hidrodestilação em aparelho de Clevenger obteve-se um teor de $0,5 \mathrm{~mL}$ e ocorre estabilização do volume extraído (Oliveira et al., 2012).

O rendimento obtido no experimento $(4,6 \%)$ ficou acima do relatado na literaura, onde autores citam rendimento médio de 1,5 a $3,5 \%$ de óleo essencial de patchouli quando extraído pelo método de hidrodestilação (Chaves et al., 2011; EPAGRI, 2004; Joy et al., 2001). As diferenças observadas no rendimento de óleo essencial podem estar relcionadas a diversos fatores, como colheita, secagem, processamento, método de extração, entre outros.

Donelian et al. (2009) relataram em seu estudo que a extração por fluido supercritico proporciona melhor rendimento de óleo essential de patchouli (5.07\%), em comparação ao método da hidrodestilação por 120 min (1.50\%). Esses resultados também obervados por Araujo (2008), que obteve em média $4,5 \%$ de óleo essencial de patchouli com o uso do método de extração supercritica. Estas diferenças no rendimento de óleo essencial e nas concentrações dos compostos nos óleos obtidos usando os dois métodos de extração podem ser atribuídas à elevada temperatura utilizada na hidrodestilação (cerca de $100^{\circ} \mathrm{C}$ ) que pode ocasionar a degradação de compostos termicamente lábeis, resultando na formação de compostos indesejáveis (Roy et al., 1996).

Masetto et al. (2007) relatam a influência do tempo de secagem no rendimento do óleo essencial de pacthouli. Os autores obtiveram maiores médias de oleo essencial de patchouli $\left(36,69 \mu \mathrm{l} \mathrm{g}^{-1}\right)$ quando a extração foi feita com 10 dias de secagem após a colheita, a temperatura ambiente, quando o tecido foliar apresentava aproximadamente 18\% de umidade, metodologia que foi adotada para a secagem do material neste trabalho. Araujo (2008) relatou em seus estudos variação no rendimento do óleo essencial de acordo com a época de colheita do patchouli no município de Florianópolis - SC, obtendo maiores rendimentos $(6 \%)$ no inverno,

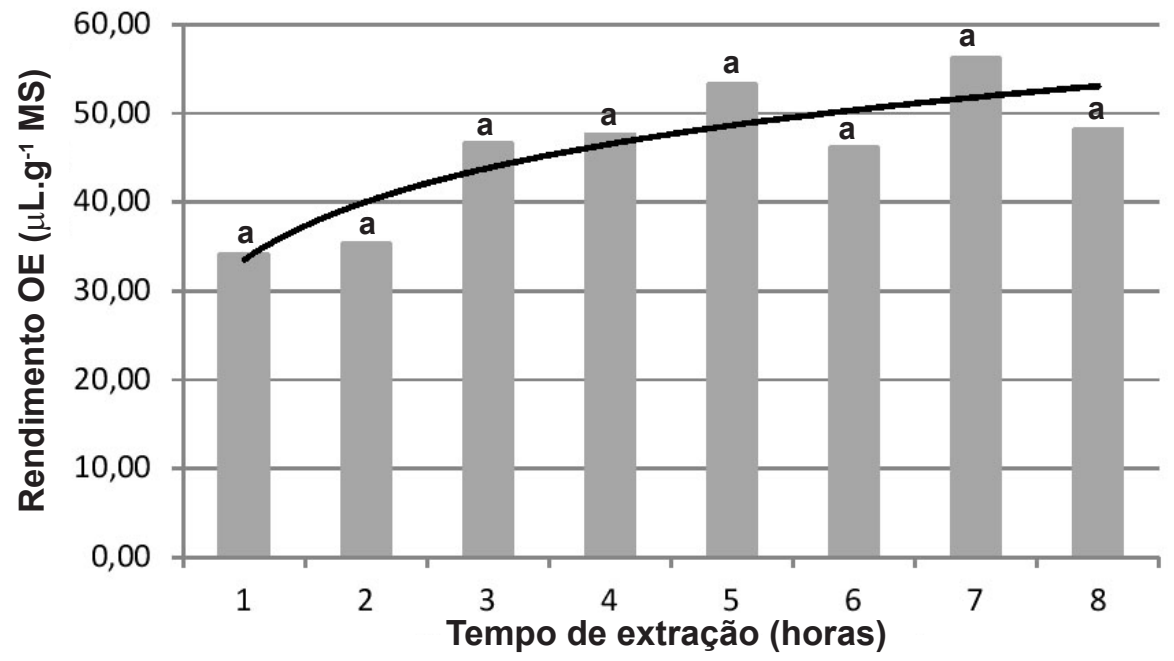

FIGURA 1. Rendimento do óleo essencial de patchouli em função dos diferentes tempos de extração $\left(R^{2}=\right.$ 0,7308). Curitiba-PR, 2006.

Rev. Bras. PI. Med., Campinas, v.15, n.3, p.319-324, 2013. 
fator que pode ter influenciado o rendimento neste trabalho, visto que as plantas foram cultivadas e colhidas em condições similares. Outro fator relatado pelo autor é o tamanho da partícula. Em seus estudos foram avaliados três tamanhos "médios" de partículas características $(0,172 ; 0,346$ e $0,555 \mathrm{~mm}$ ), observando que o tamanho "médio" de partícula ideal de patchouli é de 0,346 $\mathrm{mm}$ que proporcionou uma extração muito eficiente (aprox. $4,5 \%$ de rendimento).

A análise química das amostras do óleo essencial de patchouli apresentou, nos diferentes tratamentos, os constituintes (Tabela 1): betaguaieno $(0,49 \%$ a $0,81 \%)$, beta-patchouleno $(0,65 \%$ a $1,26 \%)$, seicheleno $(0,63 \%$ a $1,09 \%)$, alfa-selineno $(0,76 \%$ a $1,37 \%)$, cariofileno $(1,33 \%$ a $2,44 \%)$, alfa-patchouleno (1,79\% a 3,08\%), gamapatchouleno $(2,93 \%$ a $4,82 \%)$, alfa-guaieno $(3,67 \%$ a $6,48 \%)$, pogostol $(4,20 \%$ a $5,11 \%)$, alfa-bulneseno (5,01\% a $8,99 \%)$ e patchoulol $(55,74 \%$ a $64,81 \%)$. O patchoulol foi o composto majoritário, também relatado em trabalhos semelhantes (Blank et al., 2011; Wei \& Shibamoto, 2007; Buré \& Sellier, 2004; Zhu et al., 2003; Singh et al., 2002).

As porcentagens relativas dos constituintes diferem daquelas estabelecidas pela ISO (3757:2002). Elementos como o alfa-guaieno, betapatchouleno, pogostol e alfa-bulneseno estão abaixo do citado na ISO, enquanto o patchoulol apresentou porcentagens superiores. Contudo, diferenças entre os valores obtidos e aqueles estabelecidos pela ISO também foram observadas por outros autores (Chaves et al. 2011; Buré \& Sellier, 2004). De acordo com Singh et al. (2002), a qualidade do óleo essencial de patchouli é aceitável pelo comércio desde que apresente níveis entre 50.7 a $54.3 \%$ de patchoulol. Os valores encontrados neste trabalho e por Blank et al. (2011) foram superiores e seriam competitivos no mercado dos óleos essenciais.

Quanto à análise estatística dos constituintes, houve diferença significativa para a maioria dos constituintes, com exceção do seicheleno, alfa-guaieno, alfa-bulneseno e do patchoulol. As maiores porcentagens relativas dos constituintes beta-guaieno, beta-patchouleno, alfa-selineno, cariofileno, alfa-patchouleno e gamapatchouleno foram obtidas com o aumento do tempo de extração, variando entre seis e sete horas de extração. Por outro lado, o aumento do tempo de extração reduziu a quantidade de pogostol, cujas maiores porcentagens foram obtidas com duas e três horas de extração.

Araujo (2008) estudou o tempo (40, 80, $120,160,200,240,280,320$ e 360 minutos) de extração supercrítica na qualidade do óleo essencial de patchouli, observou que para os dois compostos majoritários obtidos (patchoulol e á-bulneseno) houve picos de concentração no início do processo, justificando que o óleo essencial está mais acessível e, sendo a molécula destes compostos apolar, estes são extraídos primeiro, devido à afinidade com o dióxido de carbono. Observando também um pico no final de extração, que é explicado pelo autor como um óleo essencial menos acessível, evidentemente, levando mais tempo para ser extraído (Doneliana et al., 2009).

A diferença encontrada entre as porcentagens relativas dos constituintes pode estar

TABELA 1. Médias dos constituintes do óleo essencial de patchouli (Pogostemon cablin) em função dos diferentes tempos de extração. Curitiba-PR, 2006.

\begin{tabular}{lllllllll}
\hline Constituintes (\%) & \multicolumn{7}{c}{ Tempos de extração (horas) } \\
\cline { 2 - 9 } & $\mathbf{1}$ & $\mathbf{2}$ & $\mathbf{3}$ & $\mathbf{4}$ & $\mathbf{5}$ & $\mathbf{6}$ & $\mathbf{7}$ & $\mathbf{8}$ \\
\hline Beta-guaieno & $0,50 \mathrm{bc}$ & $0,49 \mathrm{c}$ & $0,52 \mathrm{bc}$ & $0,69 \mathrm{abc}$ & $0,73 \mathrm{abc}$ & $0,81 \mathrm{a}$ & $0,80 \mathrm{a}$ & $0,78 \mathrm{ab}$ \\
Beta-Patchouleno & $0,68 \mathrm{~b}$ & $0,65 \mathrm{~b}$ & $0,75 \mathrm{~b}$ & $0,94 \mathrm{ab}$ & $0,95 \mathrm{ab}$ & $1,26 \mathrm{a}$ & $0,98 \mathrm{ab}$ & $1,00 \mathrm{ab}$ \\
Seicheleno & $0,67 \mathrm{a}$ & $0,63 \mathrm{a}$ & $0,74 \mathrm{a}$ & $0,86 \mathrm{a}$ & $0,93 \mathrm{a}$ & $1,09 \mathrm{a}$ & $0,94 \mathrm{a}$ & $0,95 \mathrm{a}$ \\
Alfa-selineno & $0,86 \mathrm{ab}$ & $0,76 \mathrm{~b}$ & $0,88 \mathrm{ab}$ & $1,13 \mathrm{ab}$ & $1,22 \mathrm{ab}$ & $1,37 \mathrm{a}$ & $1,35 \mathrm{a}$ & $1,30 \mathrm{ab}$ \\
Cariofileno & $1,43 \mathrm{ab}$ & $1,33 \mathrm{~b}$ & $1,52 \mathrm{ab}$ & $2,02 \mathrm{ab}$ & $2,15 \mathrm{ab}$ & $2,44 \mathrm{a}$ & $2,25 \mathrm{ab}$ & $2,21 \mathrm{ab}$ \\
Alfa-patchouleno & $1,95 \mathrm{bc}$ & $1,79 \mathrm{c}$ & $1,96 \mathrm{bc}$ & $2,60 \mathrm{abc}$ & $2,73 \mathrm{abc}$ & $3,08 \mathrm{a}$ & $2,98 \mathrm{a}$ & $2,92 \mathrm{a}$ \\
Gama-patchouleno & $2,93 \mathrm{bc}$ & $2,80 \mathrm{c}$ & $3,14 \mathrm{bc}$ & $4,03 \mathrm{abc}$ & $4,25 \mathrm{abc}$ & $4,82 \mathrm{a}$ & $4,45 \mathrm{ab}$ & $4,40 \mathrm{abc}$ \\
Alfa-guaieno & $4,39 \mathrm{a}$ & $3,67 \mathrm{a}$ & $4,20 \mathrm{a}$ & $5,42 \mathrm{a}$ & $5,82 \mathrm{a}$ & $6,48 \mathrm{a}$ & $6,38 \mathrm{a}$ & $6,29 \mathrm{a}$ \\
Pogostol & $4,85 \mathrm{ab}$ & $5,05 \mathrm{a}$ & $5,11 \mathrm{a}$ & $4,67 \mathrm{ab}$ & $4,70 \mathrm{ab}$ & $4,35 \mathrm{ab}$ & $4,20 \mathrm{~b}$ & $4,47 \mathrm{ab}$ \\
Alfa-Bulneseno & $5,64 \mathrm{a}$ & $5,01 \mathrm{a}$ & $5,74 \mathrm{a}$ & $7,23 \mathrm{a}$ & $7,90 \mathrm{a}$ & $8,99 \mathrm{a}$ & $8,71 \mathrm{a}$ & $8,40 \mathrm{a}$ \\
Patchoulol & $64,59 \mathrm{a}$ & $63,26 \mathrm{a}$ & $64,81 \mathrm{a}$ & $60,93 \mathrm{a}$ & $58,34 \mathrm{a}$ & $55,74 \mathrm{a}$ & $56,99 \mathrm{a}$ & $57,19 \mathrm{a}$ \\
\hline
\end{tabular}

Médias seguidas por letras minúsculas distintas diferem entre si nas linhas ao nível de $5 \%$ pelo teste de Tukey.

Fonte: Laboratório de análises de combustíveis automotivos (LACAUT)/DEQ/UFPR, 2006.

Rev. Bras. PI. Med., Campinas, v.15, n.3, p.319-324, 2013. 
relacionada ao fato destes serem sintetizados a partir de precursores comuns, onde há uma competição pelo substrato por parte dos constituintes. Como por exemplo, a redução do pogostol e o aumento do alfa-bulneseno podem ocorrer devido ao fato de possuírem como precursor comum o cátion guaianil, que em presença de água favorece a síntese do pogostol. Porém as diferenças observadas nos diferentes tempos de extração sugere que pode haver modificações ou degradação durante o processo. A estabilidade de patchoulol e pogostone foram determinadas através da injeção de solução padrão a 0 h, 1 h, 2 h, 4 h, 8 h, e 12 h após a extração. Assim, a quantificação de componentes, tais como patchoulol e pogostone em patchouli poderiam ser pré-formadas dentro de $12 \mathrm{~h}$ após a extração da amostra (Hu et al., 2006). Deguerry et al. (2006) compararam os sesquiterpenos contidos em uma amostra comercial de óleo essencial de patchouli com os sesquiterpenos encontrados em folhas frescas e sugeriram que muitos constituintes podem ser perdidos ou então incrementados durante o processo de extração. Altas porcentagens de trans-beta-cariofileno, gama-curcumeno e germacreno $D$ encontradas nos extratos das folhas frescas não foram observadas no óleo comercial. Em contrapartida, alfa-guaieno, seicheleno e alfabulneseno foram encontrados duas ou mais vezes concentrados no óleo comercial comparado ao extrato das folhas.

Outro fato que pode ter influenciado os resultados foram as condições de manejo da cultura. Quando comparamos os resultados obtidos com os estabelecidos pela ISO devemos levar em consideração que as plantas avaliadas não se encontravam nas mesmas condições climáticas e de manejo, o que poderia justificar as diferenças. Hu et al. (2006) avaliaram 18 acessos coletado de diferentes regiões da Chinas, nos quais observaram 10 compostos que estavam sempre presente porém suas concentrações variaram, demostrando a existência de três quimiotipos diferentes, o tipo patchoulol, o pogostone, e um tipo intermediário, que ficou entre os dois outros quimiotipos. Essa divisão para a espécie também foi verificada por Luo et al. (2003) e Blank et al. (2011). Neste ultimo trabalho os autores dividiram os nove acessos estudados em duas categorias, de acordo com a similaridade de seus constituintes, verificando assim um grupo caracterizado pela predominância do $\alpha$-pineno e baixa concentração de patchoulol e um segundo grupo onde predominou patchoulol e o $\alpha$-pineno esteve ausente nos quatro períodos de colheita.

Prins et al. (2006) observaram que a concentração dos compostos majoritários $\alpha$-pineno e $\beta$-mirceno no óleo essencial de alecrim $(R$. officinalis) foram favorecidos significativamente nos tempos de extração com 90 e 120 minutos em relação aos tempos de 30 e 60 minutos. A hidrodifusão favorece a extração de compostos oxigenados no início da hidrodestilação devido a maior solubilidade deste, porém com o progressivo domínio da volatilidade sobre a hidrodifusão, há uma tendência dos compostos oxigenados apresentarem relativa redução em função do aumento do teor dos compostos mais voláteis, como $\alpha$-pineno e $\beta$-mirceno (Prins et al. 2006).

O estudo indica que a extração do óleo essencial de patchouli pode ser realizada com uma hora de extração pelo método de hidrodestilação. E que o tempo de extração aumenta as porcentagens relativas do beta-guaieno, beta-patchouleno, alfaselineno, cariofileno, alfa-patchouleno e gamapatchouleno, enquanto há uma redução no teor de pogostol. Os constituintes patchoulol, alfa-guaieno, alfa-bulneseno e seicheleno, por sua vez, não alteram suas concentrações com o aumento no tempo de extração.

\section{REFERÊNCIA}

ARAUJO, A.C. Fracionamento do óleo essencial de patchouli [Pogostemon cablin (Blanco) Benth.] obtido por extração supercrítica. 2008. 108p. Dissertação (Mestrado - Desenvolvimento de Processos Químicos e Biotecnológico) - Engenharia Química, Universidade Federal de Santa Catarina, Florianópolis.

BIZZO, H.R.; HOVELL, A.M.C.; REZENDE, C.M. Óleos essenciais no Brasil: aspectos gerais, desenvolvimento e perspectivas. Química Nova, v.32, n.3, p. 588-594, 2009.

BLANK, A.F. et al. Chemical characterization of the essential oil from patchouli accessions harvested over four seasons. Industrial Crops and Products, v. 34, n. 1, p. 831-837, 2011

BURÉ, C.M.; SELLIER, N.M. Analysis of the essential oil of Indonesian patchouli (Pogostemon cablin Benth.) using GC/MS (El/Cl). Journal of Essential Oil Research, Carol Stream, v. 16, n. 1, p. 17-19, 2004.

CHAVES, F.F.M. et al. Teor e caracterização química do óleo essencial de Pogostemon cablin Benth. In: SIMPÓSIO BRASILEIRO DE ÓLEOS ESSENCIAIS, 6., 2011, Campinas. Livro de resumos. Campinas: Unicamp, 2011. p. 223.

CRAVEIRO, A.A. et al. Óleos essenciais de plantas do nordeste. Fortaleza: UFC-Departamento de Química Orgânica e Inorgânica, 1981. 210 p.

DEGUERRY, F. et al. The diverse sesquiterpene profile of patchouli, Pogostemon cablin, is correlated with a limited number of sesquiterpene synthases. Archives of Biochemistry and Biophysics, New York, v. 37, p. 123-136, 2006.

DONELIANA, A. et al. Comparison of extraction of patchouli (Pogostemon cablin) essential oil with supercritical $\mathrm{CO}_{2}$ and by steam distillation. Review Article. The Journal of Supercritical Fluids, v. 48, n. 1, p. 15-20, 2009.

EHLERT, P.A.D. et al. Tempo de hidrodestilação na

Rev. Bras. PI. Med., Campinas, v.15, n.3, p.319-324, 2013. 
extração de óleo essencial de sete espécies de plantas medicinais. Revista Brasileira de Plantas Medicinais, Botucatu, v. 8, n. 2, p. 79-80, 2006.

EPAGRI. Normas técnicas para cultivo de capim-limão, citronela, palma-rosa e patchouli. Florianópolis, 2004. 58 p. (Sistemas de Produção, 37).

HU, L.F. et al. GC-MS fingerprint of Pogostemon cablin in China. Journal of Pharmaceutical and Biomedical Analysis, v. 42, n. 2, p. 200-206, 2006.

JOY, P.P. et al. Aromatic plants. Tropical Horticulture, v. 2. (eds. Bose, T.K., Kabir, J., Das, P. and Joy, P.P.). Naya Prokash, Calcutta, p. 633-733, 2001.

KHARE, C. P. Indian Medicinal Plants: an illustrated dictionary, Springer: New Delhi, 2007. 900p.

LUO, J.P. et al. Two chemotypes of Pogostemon cablin and influence of region of cultivation and harvesting time on volatile oil composition. Acta pharmaceutica Sinica, v.38, p. 307-310, 2003.

MASETTO, M.A.M. et al. Rendimento e qualidade do óleo essencial de Pogostemon cablin Benth em diferentes períodos de secagem. 2007. In: IV Simpósio Brasileiro de Óleos Essenciais - IVSBOE (CD Simpósio). Anais... IV Simpósio Brasileiro de Óleos Essenciais. Disponível em: <http://www.ivsboe.padetec.ufc.br/CDSimposio/ quimicaeatividadesbiologicasdosoleosessenciais/ Resumo_MasettoMAM.pdf>. Acesso em: 31/07/2012

MILCHARD, M.J. et al. Application of Gas-Liquid Chromatography to the analysis of essential oils. Fingerprints of 12 essential oils. Perfumer \& Flavorist, v.29, n.5, p. 28-36, 2004.

OLIVEIRA, A.R.M.F. et al. Determinação do tempo de hidrodestilação e do horário de colheita no óleo essencial de menta. Horticultura Brasileira, v. 30, n.1, p.155-159, 2012.

PAVELA, R. Insecticidal activity of some essential oils against larvae of Spodoptera littoralis. Fitoterapia, v.76, n.7-8, p. 691-696, 2005.

PRINS, C.L.; LEMOS, C.S.; FREITAS, S.P. Efeito do tempo de extração sobre a composição e o rendimento do óleo essencial de alecrim (Rosmarinus officinalis).
Revista Brasileira de Plantas Medicinais, Botucatu, v. 8, n. 4, p. 92-95. 2006.

ROY, B.C. et al. Supercritical $\mathrm{CO}_{2}$ extraction of essential oils and cuticular waxes from peppermint leaves. Journal of Chemical Technology and Biotechnology, v. 67, n. 1, p. 21-26, 1996.

SALERNO, A.R.; REBELO, A.M.; SILVA JUNIOR, A.A. Plantas aromáticas para cultivo em Santa Catarina. Agropecuária Catarinense, v.17, n.2, p.46-49, 2004.

SILVA, L.V. et al. Extração do óleo essencial de pimenta rosa (Schinus molle) usando hidrodestilação e soxhlet. In: VI CONGRESSO BRASILEIRO DE ENGENHARIAQUÍMICA EM INICIAÇÃO CIENTÍFICA - COBEQ - IC, 2005, Foz do Iguaçu. Anais... VI Congresso Brasileiro de Engenharia química em iniciação científica. 2005 p. 1-7.

SINGH, M.; SHARMA, S.; RAMESH, S. Herbage, oil yield and oil quality of patchouli [Pogostemon cablin (Blanco) Beth.] influenced by irrigation, organic mulch and nitrogen application in semi-arid tropical climate. Industrial Crops and Products, Amsterdam, v. 16, p. 101-107, 2002.

SOUZA, W.P. et al. Avaliação do teor e da composição química de óleo essencial de Mentha piperita (L.) Huds durante o período diurno em cultivo hidropônico. Revista Brasileira de Plantas Medicinais, v.8, p. 108-111. 2006

VALMORBIDA, J. et al. Rendimento e composição química de óleos essenciais de Mentha x piperita L. cultivada em solução nutritiva com diferentes concentrações de potássio. Revista Brasileira de Plantas Medicinais, v.8, p.56-61, 2006.

ZHU, B.C.R. et al. Toxicity and repellency of patchouli oil and patchouli alcohol against formosan subterranean termites Coptotermes formosanus Shiraki (Isoptera: Rhinotermitidae). Journal of Agricultural And Food Chemistry, v.51, p.4585-4588, 2003.

WEI, A.; SHIBAMOTO, T. Antioxidant activities and volatile constituents of various essential oils. Journal of Agricultural And Food Chemistry, v.55, n.5, p.17371742, 2007.

Rev. Bras. PI. Med., Campinas, v.15, n.3, p.319-324, 2013. 